\title{
SEISMIC BEHAVIOR OF INTAKE TOWER OF DAM
}

\author{
Chethan kumar B ${ }^{1}$, M.R Suresh ${ }^{2}$, Ravikumar HS ${ }^{3}$, B Sivakumara swamy ${ }^{4}$ \\ ${ }^{1}$ Postgraduate student, Structural engineering, Dept of Civil engineering, Dr AIT Bangalore \\ ${ }^{2}$ Assistant professor, Geotechnical engineering, Dept of Civil engineering, Dr AIT Bangalore \\ ${ }^{3}$ Assistant professor, Structural engineering, Dept of Civil engineering MSRIT, Bangalore \\ ${ }^{4}$ Professor \& Head of the Dept of Civil engineering, Dr AIT Bangalore
}

chethan.1911@gmail.com

\begin{abstract}
Intake towers are typically tall, hollow, reinforced concrete structures and form entrance to reservoir outlet works. A parametric study on dynamic behavior of circular cylindrical towers can be carried out to study the effect of depth of submergence, wall thickness and slenderness ratio, and also effect on tower considering dynamic analysis for time history function of different soil condition and by Goyal and Chopra accounting interaction effects of added hydrodynamic mass of surrounding and inside water in intake tower of dam
\end{abstract}

Keywords: Hydrodynamic mass, Depth of submergence, Reservoir, Time history analysis,

\section{INTRODUCTION}

Intake towers are typically tall, hollow reinforced concrete structures and form entrance to reservoir outlet works. They often house equipment for regulating the release of impounded water for vital public services such as water supply or generation of electricity, aid in emptying the reservoir in an emergency condition, and permit reservoir lowering for inspections and special repairs. Intake structures can be located within or attached to concrete gravity dams or located outside the dam.

\subsection{Problem Description}

The dynamic response of an intake tower during an earthquake may present quite complex characteristics due to many factors. The water, inside and outside of the tower, plays an important role in the modification of the response of the structure. The behavior of the structure to the earthquake loading is very important considering the various benefits of the intake towers. Also IS codes does not provide any information for the dynamic analysis of the intake towers, so it is necessary to understand the dynamic behavior of these towers.

Modeling and analysis of intake towers requires sound understanding of the behavior due to the complexities involved. The intake tower having axis symmetric geometry and being submerged in an unbounded reservoir necessitates a 3-dimensional model for accurate analysis. It is important to incorporate fluid-structure-interaction and the effect of unbounded reservoir condition while carrying out a seismic analysis. Thus it is necessary to investigate suitable method of analysis and modeling techniques to know the dynamic behavior intake towers.

\subsection{Objective}

To study the different parameter that considerably affects the behavior of intake towers during the event of earthquake. The following parameters have been considered for the study

1. Depth of submergence $\left(H_{o} / H_{i}\right)$

2. Outside $\operatorname{radius}\left(r_{o}\right)$ to height $(H)$ of tower, i.e., $r_{o} / H$

3. Wall thickness $(t)$

4. Direction of ground motion on response of tower.

Simplified model of a uniform and non-uniform intake tower is considered for the analysis in SAP 2000. Hydrodynamic effects were included in the analysis using the concept of hydrodynamic added masses (Goyal and Chopra 1989).

\section{MODEL OF INTAKE TOWER}

A rectangular tower (Figure 1) of height of structure $61 \mathrm{~m}$, the depth of surrounding and inside water for all the models is taken equal to the height of the structure. Modal analysis is carried in SAP2000 to obtain the dynamic properties of the tower structure 


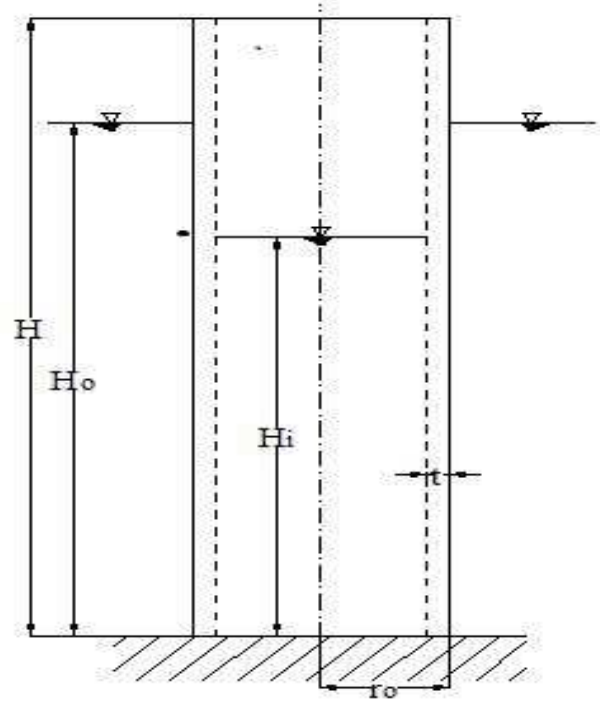

Figure 1 Typical intake tower

\section{CALCULATION OF HYDRODYNAMIC MASS}

The fluid structure interaction is considered by calculating hydrodynamic mass of inside and outside water by the simplified procedure for evaluating the added hydrodynamic mass that is accurate enough for preliminary earthquake analysis of towers developed (Goyal and Chopra 1989) is mentioned below.

According to this method, the mass of an intake tower $m_{s}(z)$ having an arbitrary cross section with two axes of symmetry is replaced the by the virtual mass $\widetilde{\mathrm{m}}_{\mathrm{s}}(\mathrm{z})$ as:

$\widetilde{\mathrm{m}}_{\mathrm{s}}(\mathrm{z})=\mathrm{m}_{\mathrm{s}}(\mathrm{z})+\mathrm{m}_{\mathrm{a}}^{\mathrm{o}}(\mathrm{z})+\mathrm{m}_{\mathrm{a}}^{\mathrm{i}}(\mathrm{z})$

Here, the added hydrodynamic masses $\mathrm{m}_{\mathrm{a}}^{\mathrm{o}}(\mathrm{z})$ and $\mathrm{m}_{\mathrm{a}}^{\mathrm{i}}(\mathrm{z})$ represent the effects of the outside and inside water, respectively, on the dynamic response of the tower.

\section{DYNAMIC ANALYSIS}

\subsection{Model Analysis:}

Model analysis has been carried out by considering ritz vector because the Ritz vectors yield excellent results is that they are generated by taking into account the spatial distribution of the dynamic loading, whereas the direct use of the natural mode shapes neglects this very important information.

By considering 12 natural modes and starting Load Vectors as acceleration in $\mathrm{x}$ direction model analysis of fully submerged, half submerged, quarter submerged \& no water conditioned towers has been analyzed (figure 4 )
Time history analysis of the intake tower has been carried by considering Time history function of recent earthquake India from 2005 to 2013 for different soil condition such as medium and hard soil condition of different seismic zones, which will give us critical response of the structure (figure 5)

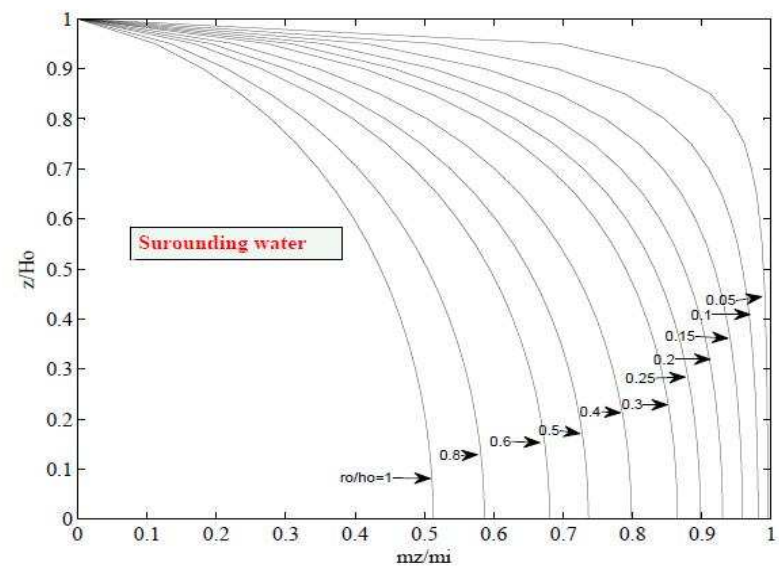

Figure 2 Calculation of surrounding water for equation 3.1

The above graphs has been plotted by considering concept given by Chopra and Goyal by using MATLAB program

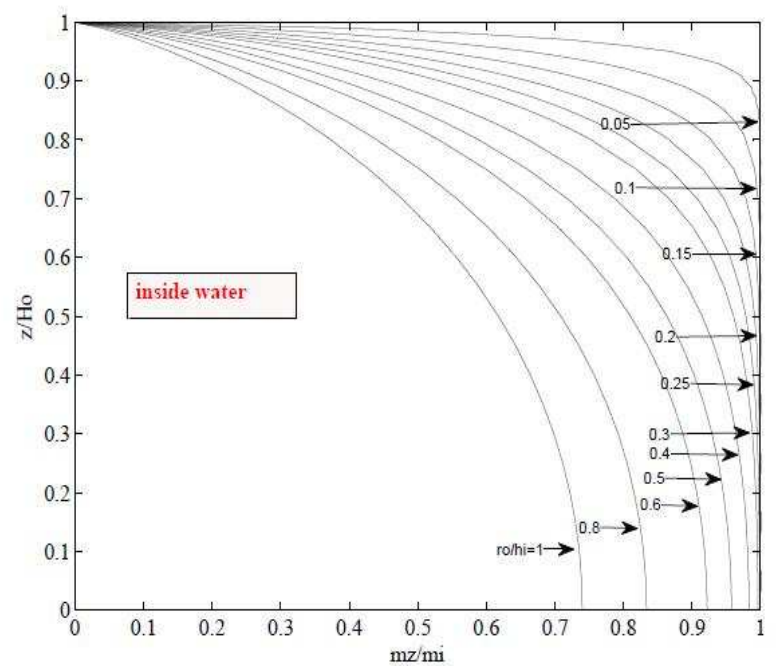

Figure 3 calculation of surrounding water for equation 3.1

\subsection{Response Spectrum Analysis:}




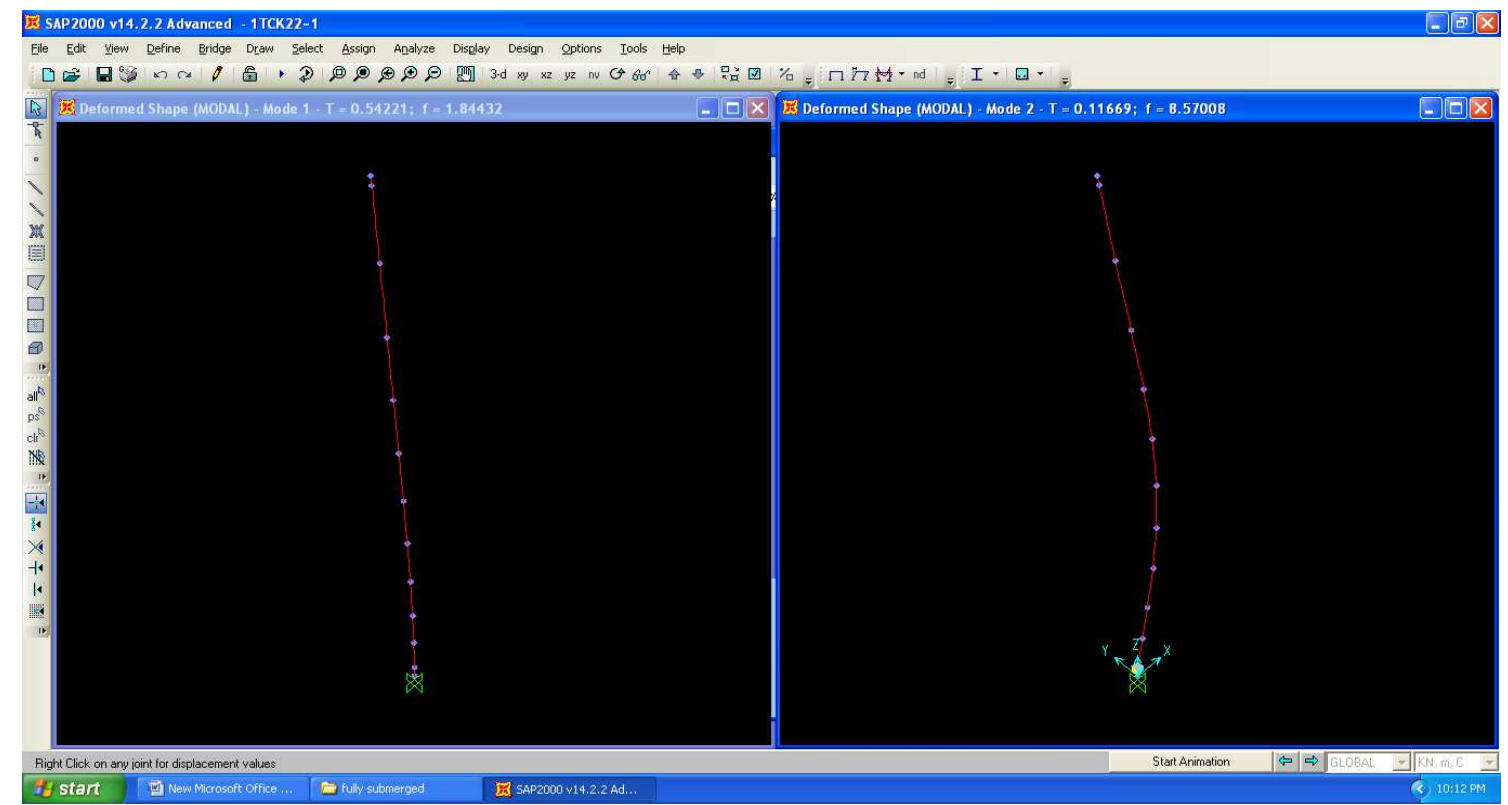

Figure 4 Model analysis in fully submerged condition of water

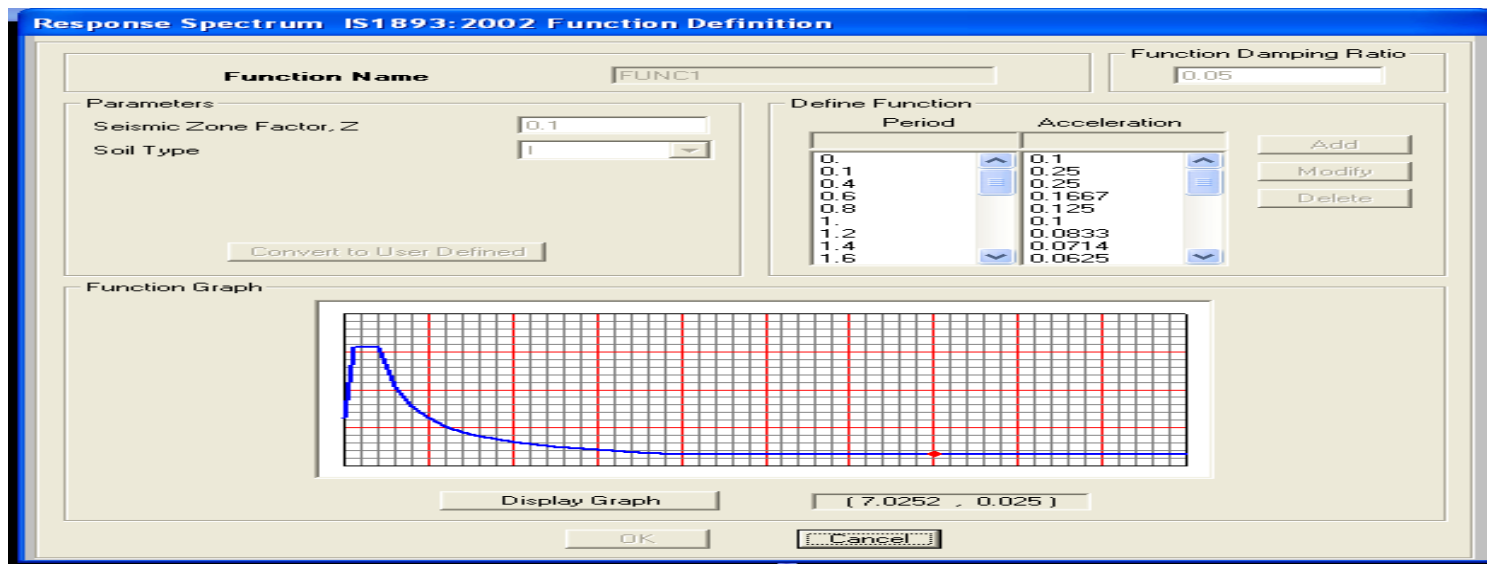

Figure5 response spectrum curve for hard soil in zone 2

\section{RESULTS}

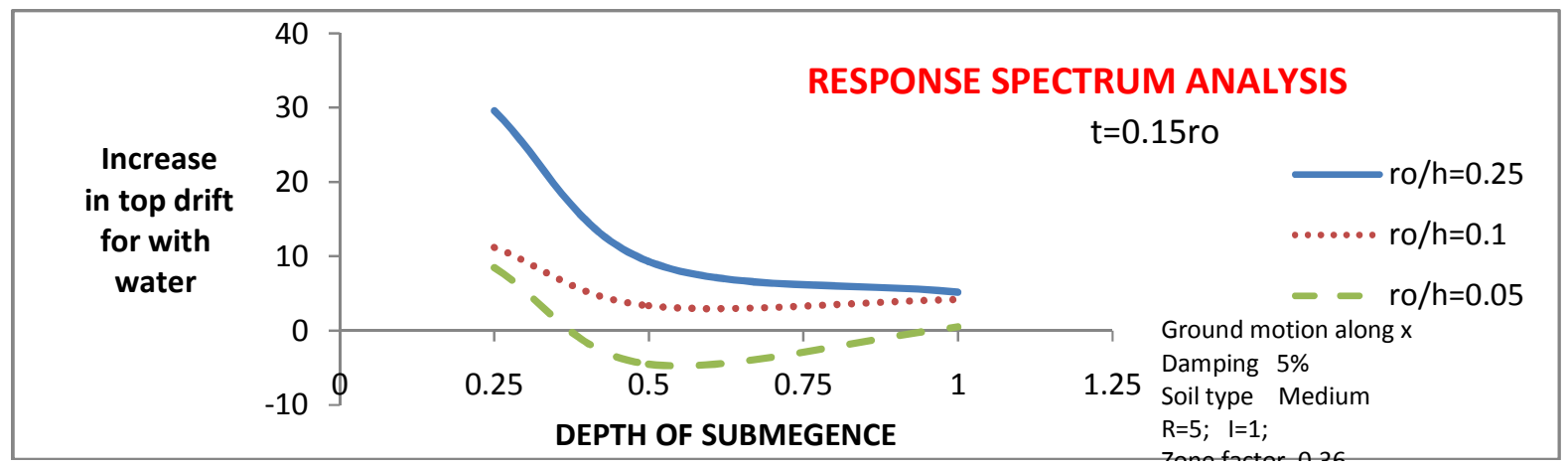



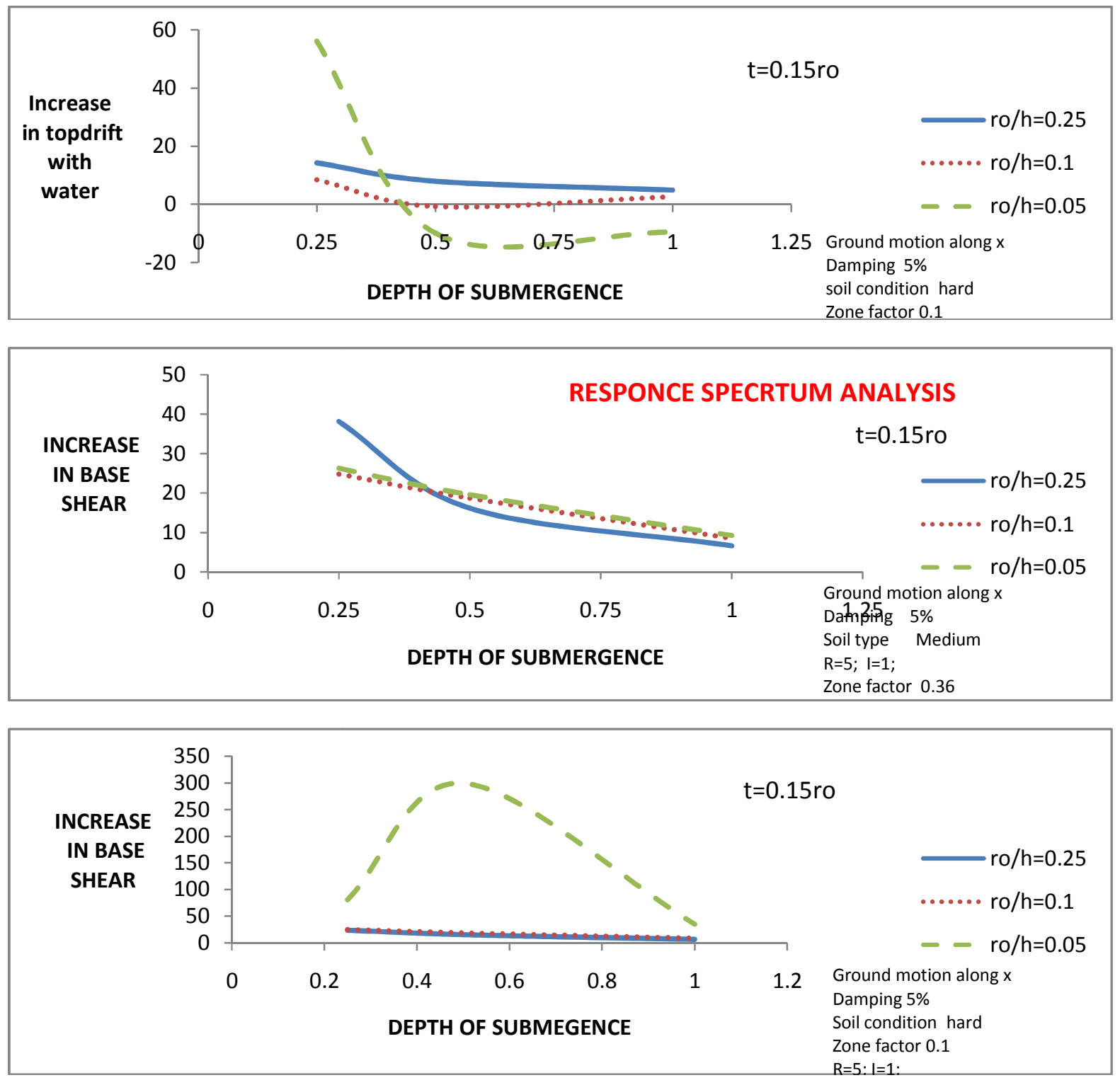

\section{REFERENCES}

[1] Goyal, A., and Chopra, A. K. (1989). "Hydrodynamic and foundation interaction effects in dynamics of intake towers: earthquake responses," / . Struct. Engrg., ASCE, 115(4), 1386-1395.

[2] Liaw, C-Y., and Chopra, A. K. (1974). "Dynamics of towers surrounded by water." Int. J. of Earthquake Engrg. and Struct. Dynamics, 3(1), 33-49.

[3] Liaw, C-Y., and Chopra, A. K. (1975). "Earthquake analysis of axisymmetric towers partially submerged in water." J. of Earthquake Engrg. and Struct. Dyn., 3(3), 233-248 